$12-1-2008$

\title{
Scattering of an Electromagnetic Plane Wave by a Luneburg Lens. I. Ray Theory
}

James A. Lock

Cleveland State University, j.lock@csuohio.edu

Follow this and additional works at: https://engagedscholarship.csuohio.edu/sciphysics_facpub

Part of the Physics Commons

How does access to this work benefit you? Let us know!

\section{Publisher's Statement}

This paper was published in Journal of the Optical Society of America A: Optics Image Science and Vision and is made available as an electronic reprint with the permission of OSA. The paper can be found at the following URL on the OSA website: http://www.opticsinfobase.org/josaa/ abstract.cfm?URI=josaa-25-12-2971. Systematic or multiple reproduction or distribution to multiple locations via electronic or other means is prohibited and is subject to penalties under law.

\section{Original Citation}

Lock, James A. "Scattering of an Electromagnetic Plane Wave by a Luneburg Lens. I. Ray Theory." Journal of the Optical Society of America A: Optics Image Science and Vision 25 (2008): 2971-2979.

\section{Repository Citation}

Lock, James A., "Scattering of an Electromagnetic Plane Wave by a Luneburg Lens. I. Ray Theory" (2008). Physics Faculty Publications. 30.

https://engagedscholarship.csuohio.edu/sciphysics_facpub/30

This Article is brought to you for free and open access by the Physics Department at EngagedScholarship@CSU. It has been accepted for inclusion in Physics Faculty Publications by an authorized administrator of EngagedScholarship@CSU. For more information, please contact library.es@csuohio.edu. 


\title{
Scattering of an electromagnetic plane wave by a Luneburg lens. I. Ray theory
}

\author{
James A. Lock \\ Department of Physics, Cleveland State University, Cleveland, Ohio 44115 (j.lock@csuohio.edu)
}

Received July 30, 2008; accepted September 11, 2008;

posted September 29, 2008 (Doc. ID 99456); published November 12, 2008

\begin{abstract}
For a plane wave incident on either a Luneburg lens or a modified Luneburg lens, the magnitude and phase of the transmitted electric field are calculated as a function of the scattering angle in the context of ray theory. It is found that the ray trajectory and the scattered intensity are not uniformly convergent in the vicinity of edge ray incidence on a Luneburg lens, which corresponds to the semiclassical phenomenon of orbiting. In addition, it is found that rays transmitted through a large-focal-length modified Luneburg lens participate in a far-zone rainbow, the details of which are exactly analytically soluble in ray theory. Using these results, the Airy theory of the modified Luneburg lens is derived and compared with the Airy theory of the rainbows of a homogeneous sphere. (C) 2008 Optical Society of America

OCIS codes: $080.2710,080.5692$.
\end{abstract}

\section{INTRODUCTION}

A Luneburg lens is a dielectric sphere whose refractive index decreases from a large value at the center of the sphere to the index of the surrounding medium at its surface [1] in such a way that, in the context of ray theory, an electromagnetic plane wave incident on the lens focuses at the axial point on the shadowed side of the lens surface. A practical application of such a lens is that if a point source of radiation were placed on the sphere surface, a family of parallel rays with flat phase fronts would exit the other side of the lens and act as a highly directional outgoing beam [2-4]. A modified Luneburg lens is obtained by changing in concert both the value of the refractive index at the center of the sphere and the falloff rate so that a plane wave incident on such an inhomogeneous sphere would focus on the axis either at a point inside the sphere [5] or outside it, if it were not for spherical aberration produced as the converging rays exit the lens on their way to the exterior focal point. An alternative modified Luneburg lens, which will not be considered here, has a different radial variation of refractive index that compensates for the spherical aberration so that an incident plane wave focuses to a point outside the lens [4,6-8].

This is the first in a series of papers $[9,10]$ examining a wide range of phenomena occurring when an electromagnetic plane wave is scattered by either a Luneburg lens or a modified Luneburg lens. In particular, a number of interesting effects occur that are impossible for scattering of a plane wave by a homogeneous sphere. For example, when the lens's focal length is larger than its radius, a rainbow occurs for light transmitted through a modified Luneburg lens, whereas for scattering by a homogeneous sphere, rainbows occur only for rays making one or more internal reflections. When the lens's focal length and radius are equal, the rainbow evolves into an orbiting ray $[11,12]$. If the focal length becomes smaller than the radius, the orbiting ray evolves into a family of morphologydependent resonances (MDRs). The transmission rainbow is analyzed in this paper using both ray theory and Airy theory. In the other two papers in this series $[9,10]$ the orbiting ray is examined using the physical optics model where the wavefront exiting the Luneburg lens is Fraunhofer diffracted to the scattering far zone. In addition, both the orbiting ray and MDRs are examined (i) using the effective potential approach and (ii) approximating the continuously varying refractive index with a finely stratified multilayer sphere.

The body of this paper proceeds as follows. In Section 2 the geometry of a Luneburg lens is described. In Sections $3-5$ the ray trajectories, the scattered amplitude, and the phase are derived when the transmitted rays focus on the lens surface $(f=1)$, inside the lens $(f<1)$, and outside the lens $(f>1)$, respectively. In Section 6 the Airy theory of the transmission rainbow is developed and discussed. Finally, in Section 7 the principal results of this paper are briefly recounted.

\section{GEOMETRY}

Consider a sphere of radius $a$ centered at the origin of an $x, y, z$ coordinate system with the radially inhomogeneous refractive index profile [1]:

$$
N(r)=\left[2-(r / a)^{2}\right]^{1 / 2} .
$$

Such a sphere is called a Luneburg lens, and its refractive index profile is graphed in Fig. 1. The medium external to the Luneburg lens is assumed to be air with refractive in$\operatorname{dex} N=1$. The refractive index varies approximately parabolically for $r \ll a$, and it has a finite nonzero slope at $r$ $=a$. A family of incident rays with amplitude $E_{0}$, wavelength $\lambda$, and wave number $k=2 \pi / \lambda$ propagate in the external medium parallel to the $z$ axis from negative $z$ toward positive $z$ and are incident on the Luneburg lens, as is shown in Fig. 2(a). As the rays are transmitted through the lens, they focus at the axial point $(z, \rho)=(a, 0)$ on the shadowed side of the lens, where $\rho$ is the radial coordinate 


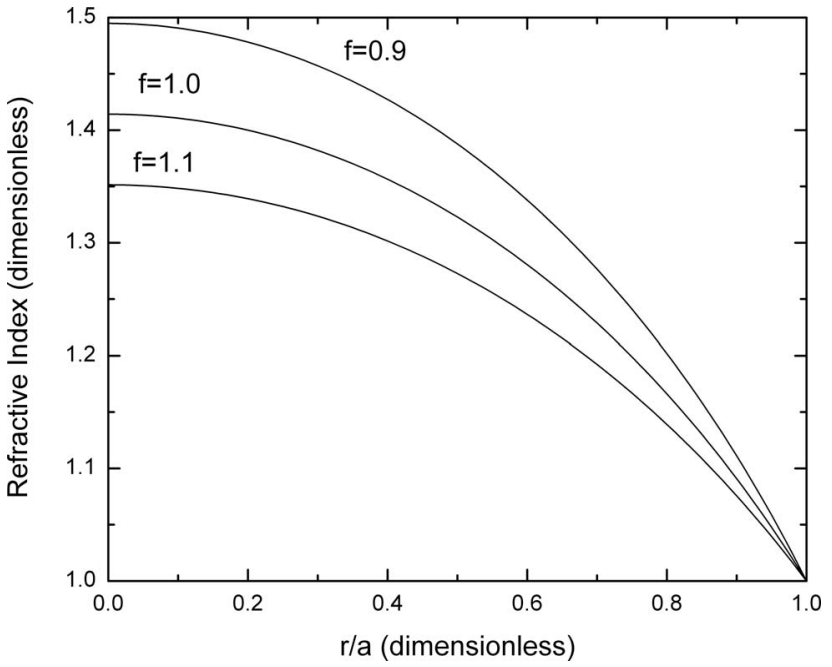

Fig. 1. Refractive index of a modified Luneburg lens as a function of $r / a$ for $f=0.9,1.0$, and 1.1.

in the $x y$ plane. For simplicity, in this paper I will refer to the ray trajectories in the $x z$ plane where the $z$ axis is horizontal and the $x$ axis is vertical. The geometry of the system in three dimensions can then be obtained by rotating Figs. 2(a)-2(c) about the $z$ axis. Since the refractive index profile of the Luneburg lens is radially inhomogeneous, the transmitted ray paths inside the lens are curved. It has been shown that each of these curved paths is a portion of an ellipse passing through the focal point and having a horizontal tangent at the point where the incoming ray enters the sphere [5].

Consider also the family of modified Luneburg lenses of radius $a$ with the refractive index profile

$$
N(r)=\left[1+f^{2}-(r / a)^{2}\right]^{1 / 2} / f .
$$

These profiles are also graphed in Fig. 1 for $f=0.9$ and 1.1 . It has been shown that each of the transmitted curved ray paths inside the lens is again a portion of an ellipse [5], and if $f<1$, the transmitted rays focus inside the lens at the axial point $(z, x)=(f a, 0)$ as is shown in Fig. 2(b). The case $f=1$ is the Luneburg lens of Eq. (1). For $f>1$, consider how the ray trajectories would look if the refractive index variation of Eq. (2) applied for $r \leqslant a$ only when $z$ $>0$ and for all space when $z \geqslant 0$. The transmitted rays would again focus at $(z, x)=(f a, 0)$. But for a spherical $f$ $>1$ modified Luneburg lens in air where Eq. (2) is valid for $z \leqslant a$ on both the illuminated and the shadowed sides of the sphere, the point focus is distorted into a near-zone spherical aberration caustic by the refraction of the converging rays as they leave the lens at $r=a$ on their way to the focal point. This behavior is suggested in Fig. 2(c).

\section{RAY SCATTERING FOR $f=1$}

\section{A. Ray Trajectories}

The transmitted ray path inside the Luneburg lens is described in terms of polar coordinates $r(\xi)$ where $\xi=0$ on the positive $z$ axis and $\xi$ increases toward the positive $x$ axis. Let $\psi$ be the angle between the radius vector and the forward propagation direction of the ray at any point on its path. (This geometry is illustrated in Fig. 3.5 of [13] with $\psi$ replaced by $\varphi$ and $\xi$ replaced by $\theta$ there.) Since ray trajectories conserve angular momentum, each ray path satisfies [13]

$$
r N(r) \sin (\psi)=b,
$$

where $b$ is a constant that will shortly be shown to be the incident ray impact parameter. The ray propagation equation in polar coordinates is then [13]

$$
\mathrm{d} r / \mathrm{d} \xi=r\left[(r N / b)^{2}-1\right]^{1 / 2} .
$$

A ray trajectory is labeled by the angle of incidence $\beta$ that an incoming ray makes with the normal to the sphere surface. Since $N(a)=1$ for both a Luneburg lens and a modified Luneburg lens, no refraction or reflection of the ray occurs at the sphere surface as it enters or leaves the lens, and the impact parameter of the ray is

$$
b=a \sin (\beta) .
$$

This impact parameter is illustrated in Fig. 4 of [14].

As was mentioned above, the path of a ray inside a Luneburg lens is a portion of an ellipse. However, the axes of that ellipse are rotated by the angle $\alpha$ with respect to the $z, x$ laboratory axes. This is evident in Figs. 2(a)-2(c) and in Figs. 1, 2, 3, 4, and 5 of [5]. Consider a second coordinate system $z^{\prime}, x^{\prime}$ rotated by this angle $\alpha$ with respect to $z x$ so that

$$
\xi^{\prime}=\xi-\alpha,
$$

and $\alpha$ is positive when $z^{\prime}$ is rotated from the $z$ axis toward the $x$ axis. The trajectory of the transmitted ray path can be straightforwardly derived in the $z^{\prime}, x^{\prime}$ coordinate system in the following way. An ellipse centered on the origin in the rotated coordinate system with its axes along the $z^{\prime}$ and $x^{\prime}$ directions is given by

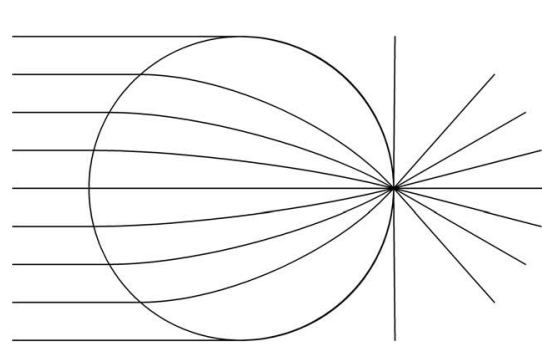

(a)

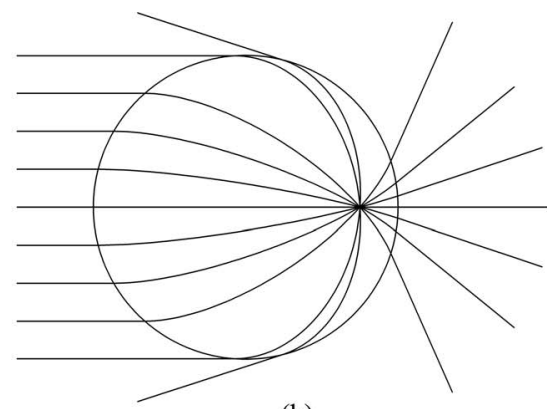

(b)

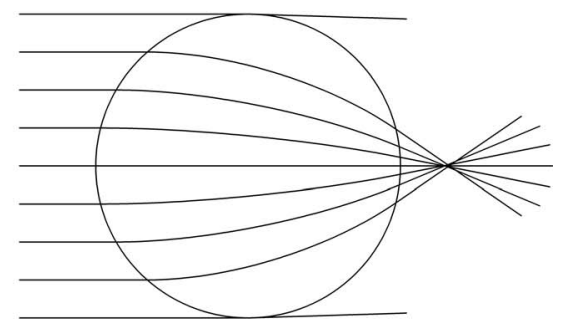

(c)

Fig. 2. Ray trajectories through a modified Luneburg lens for (a) $f=1.0$, (b) $f=0.75$, and (c) $f=1.25$. 


$$
r^{2} \cos ^{2}\left(\xi^{\prime}\right) /\left(a^{2} A\right)+r^{2} \sin ^{2}\left(\xi^{\prime}\right) /\left(a^{2} B\right)=1,
$$

where $A$ and $B$ are arbitrary. The ellipse parameters $A$ and $B$ and the rotation angle $\alpha$ of the $z^{\prime}, x^{\prime}$ axes with respect to the laboratory $z, x$ axes are determined by imposing three conditions on Eq. (7): (i) the focal point of the rays at $(z, x)=(a, 0)$ in the original coordinate system lies on the ellipse, (ii) the slope of the ellipse is zero at the point in the original coordinate system where the ray enters the lens $(z, x)=(-a \cos (\beta), a \sin (\beta))$, and (iii) the trajectory satisfies Eq. (4) for ray propagation with the refractive index profile given by Eq. (1) and the impact parameter $b$ given by Eq. (5). It is found that a positive value of $\beta$ for a ray incident on the top half of the lens corresponds to a negative value of $\alpha$. After a reasonably large amount of algebra, the ray trajectory is the ellipse of Eq. (7) with

$$
\begin{aligned}
& \beta=-2 \alpha, \\
& A=1+\cos (\beta), \\
& B=1-\cos (\beta) .
\end{aligned}
$$

The equation for the ray trajectory in the laboratory $z, x$ coordinate system expressed in terms of the polar coordinates $r, \xi$ then simplifies to

$$
r(\xi)=a \sin (\beta) /[1-\cos (\beta) \cos (2 \xi+\beta)]^{1 / 2}
$$

for $0 \leqslant \xi \leqslant \pi-\beta$. The ray enters the sphere at $\xi=\pi-\beta$ and exits at $\xi=0$. For future reference, the curved ray path reaches its closest approach to the origin at the angle

$$
\xi_{0}=(\pi-\beta) / 2,
$$

and the distance of closest approach to the origin is

$$
r_{0}=r\left(\xi_{0}\right)=a[1-\cos (\beta)]^{1 / 2} .
$$

The trajectory is symmetric about the angle $\xi_{0}$.

\section{B. Scattering Angle and Scattered Intensity}

Let the scattering angle $\theta$ be the angle between the path of a ray after it has exited the Luneburg lens and the positive $z$ axis. After a ray incident on the top half of the lens has been transmitted through it, its exit direction lies between the positive $z$ and negative $x$ axes, as is shown in Fig. 4 of [14]. Such a ray is defined to have positive $\theta$. Since each ray exits the lens at $\xi_{\text {exit }}=0$, conservation of angular momentum given by Eqs. (3) and (5) immediately yields

$$
\theta=\beta \quad \text { for } 0 \leqslant \theta \leqslant \pi / 2 .
$$

This is the simplest relation possible between an incoming ray's impact parameter and the scattering angle of the corresponding outgoing ray. As a result, it may be argued that transmission through a Luneburg lens represents the simplest case of ray scattering.

As is commonly done for scattering by a homogeneous sphere, the Luneburg lens scattered intensity $I_{\text {scat }}(\theta)$ can be easily obtained using flux conservation $[15,16]$. If the intensity of the incident plane wave is $I_{0}$ and a detector recording the scattered light is in the far zone a distance
$R$ from the origin of coordinates, flux conservation applied to the transmitted light gives

$$
I_{\text {scat }}(\theta)=\left(I_{0} a^{2} / R^{2}\right) \sin (\beta) \cos (\beta) T(\theta) /[\sin (\theta)|\mathrm{d} \theta / \mathrm{d} \beta|],
$$

which simplifies to [14]

$$
\begin{aligned}
I_{\text {scat }}(\theta) & =\left(I_{0} a^{2} / R^{2}\right) \cos (\theta) T(\theta) \quad \text { for } 0 \leqslant \theta \leqslant \pi / 2, \\
& =0 \quad \text { for } \pi / 2 \leqslant \theta \leqslant \pi,
\end{aligned}
$$

where $T(\theta)$ is the fraction of the incident light intensity transmitted through the lens. The magnitude of the scattered electric field $E_{\text {scat }}(\theta)$ is the square root of Eq. (14).

Whenever a ray encounters an interface between two different refractive indices, part of the ray amplitude is refracted, part is reflected, and the refracted ray changes its direction in accordance with Snell's law. Since the refractive index inside the Luneburg lens is a function of $r$, the continual change in direction of the transmitted ray becomes the elliptical ray path, and reflected rays are in principle produced at every point along this curved trajectory. One can show, however, that $T(\theta)=1$ in the $\lambda \rightarrow 0$ ray theory limit. The argument is outlined as follows. Since a ray trajectory in the Luneburg lens is symmetric about $\xi_{0}$, the ray encounters the same refractive index profile for $\pi-\beta \geqslant \xi \geqslant \xi_{0}$ and for $\xi_{0} \geqslant \xi \geqslant 0$. When one decomposes the Luneburg lens into a finely stratified multilayer sphere, then multiplies together all the transmission coefficients along the ray path through the multilayer sphere, and finally lets the number of layers go to infinity, one obtains $T(\theta)=1$ for both polarizations. The transmitted ray thus suffers no loss in amplitude and acquires only a phase shift as it passes through the Luneburg lens. This result relied on the fact that all the reflected rays produced at interface changes propagate in different directions. In contrast to this, $T$ is nonzero and can approach unity for a plane-parallel Bragg grating where ray propagation occurs only in one dimension and the reflected rays add coherently.

Since the scattered electric field $E_{\text {scat }}(\theta)$ is proportional to $(\cos \theta)^{1 / 2}$ as in Fig. 3, the slope of $E_{\text {scat }}$ is infinite when $\beta=\pi / 2$. This divergence is known as a weak caustic [17]. Such an effect also occurs in scattering of a plane wave by an air bubble in water at the transition from partial to total external reflection. A number of interesting scattering effects occur in the vicinity of this weak caustic. It will be shown in Subsection 4.B. and Section 5 that both the scattering angle and the scattered intensity of a modified Luneburg lens are not uniformly convergent in the vicinity of $f \rightarrow 1$ and $\beta \rightarrow \pi / 2$; i.e., the result depends on the order in which the two limits are taken. In addition, as is seen in Fig. 2(a) for $f=1$ and $\beta=\pi / 2$, the ray incident at the edge of the Luneburg lens travels in a circular arc with $r=a$ for a quarter of a revolution according to Eq. (9) and exits the lens at $\theta=\pi / 2$ according to Eq. (12). But intuitively, it would seem that there should be no special reason why such a ray traveling in a circular trajectory on the surface of the Luneburg lens must break free of the surface only at $\theta=\pi / 2$. One could argue that the ray could equally well propagate along the circumference of the lens for any number of revolutions shedding secondary rays as 


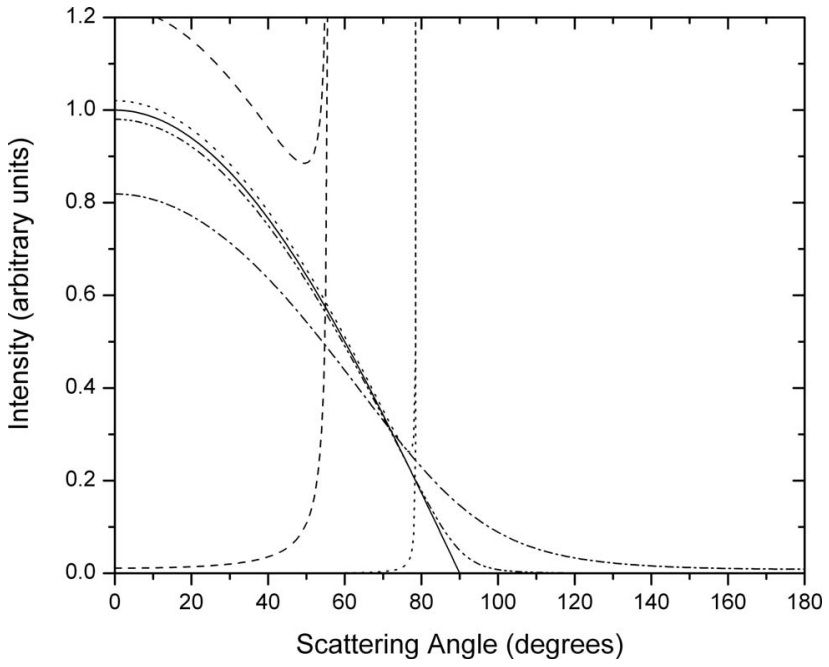

Fig. 3. Ray intensity as a function of the scattering angle $\theta$ for $f=0.90$ (dot-dashed curve), $f=0.99$ (dot-dot-dashed curve), $f=1.0$ (solid curve), $f=1.01$ (dotted curve), and $f=1.10$ (dashed curve). For $f=1.01$ the rainbow angle is $\theta_{R}=78.61^{\circ}$, and for $f=1.10$ the rainbow angle is $\theta_{R}=55.74^{\circ}$.

it does, in qualitatively much the same way that a Fock transition is associated with the shedding of electromagnetic surface waves [14]. It will be shown in [9] that the edge ray exhibits the semiclassical phenomenon of orbiting and traverses the sphere surface forever and is the source of the smooth attenuation of $I_{\text {scat }}(\theta)$ for $\theta \geqslant \pi / 2$ that is found to occur when the situation is analyzed using wave theory.

\section{Phase of the Scattered Light}

The optical path length along the curved trajectory of the ray inside the Luneburg lens is

$$
\begin{aligned}
\int N \mathrm{~d} s= & a \int_{0}^{\pi-\beta} \mathrm{d} \xi \sin (\beta)[1-2 \cos (\beta) \cos (2 \xi+\beta) \\
& \left.+\cos ^{2}(\beta)\right] /[1-\cos (\beta) \cos (2 \xi+\beta)]^{2} \\
= & a[\pi / 2+\cos (\beta)],
\end{aligned}
$$

where $\mathrm{d} s$ is the differential arc length along the trajectory. The integrand in Eq. (15) is obtained by substituting Eq. (9) for $r(\xi)$ into Eq. (1) for $N(r)$ and Eq. (4) for $\mathrm{d} r / \mathrm{d} \xi$ into the standard expression for the arc length expressed in polar coordinates. Continuing the calculation of the phase, the optical path length of an incident ray along its trajectory from the lens's entrance plane to the lens surface plus that of the associated outgoing ray from the lens surface to the lens's exit plane is $2 a[1-\cos (\theta)]$. The phase of the scattered ray is standardly written with respect to the phase of a reference ray that propagates along the negative $z$ axis to the origin, turns, and then propagates out at the scattering angle $\theta$ as if the sphere were absent. The optical path length of a ray along its trajectory from the lens's entrance plane to its exit plane with respect to that of the reference ray is then

$$
L=a[\pi / 2-\cos (\theta)] .
$$

As a check of this calculation, the optical path length from the lens's entrance plane to the focal point is

$$
L=a(1+\pi / 2),
$$

which is independent of $\beta$, producing constructive interference of all the rays at the focal point. In addition, each transmitted ray participates in the point focus as it crosses the $z$ axis, acquiring a non-path-length phase of $-\pi$ according to the van de Hulst rules [18]. Combining Eq. (14) for the amplitude of the transmitted light and Eq. (16) for the phase of the transmitted light and adding the Fraunhofer diffraction of a plane wave by a sphere of radius $a$, the total electric field in ray theory is then

$$
\begin{aligned}
E_{\text {scat }}(\theta)= & \left(E_{0} a / R\right)(\cos \theta)^{1 / 2} \exp \left\{i\left[k R+x_{L}(\pi / 2-\cos (\theta))-\pi\right]\right\} \\
& +i\left(E_{0} a / R\right) x_{L}\left[J_{1}\left(x_{L} \theta\right) /\left(x_{L} \theta\right)\right] \\
& \times \exp (i k R) \text { for } 0 \leqslant \theta \leqslant \pi / 2 \\
= & 0 \quad \text { for } \pi / 2<\theta \leqslant \pi,
\end{aligned}
$$

where the size parameter of the Luneburg lens is defined as

$$
x_{L}=2 \pi a / \lambda
$$

and $J_{1}$ is a Bessel function.

\section{RAY SCATTERING FOR $\boldsymbol{f}<\mathbf{1}$}

\section{A. Ray Trajectories}

The trajectory of a ray incident on a modified Luneburg lens is again a portion of the ellipse of Eq. (7) in the $z^{\prime}, x^{\prime}$ rotated coordinate system with the ellipse axes along the $z^{\prime}$ and $x^{\prime}$ directions. As before, the two unknown ellipse parameters $A, B$, in the $z^{\prime}, x^{\prime}$ coordinate system and the rotation angle $\alpha$ of the $z^{\prime}, x^{\prime}$ system with respect to the laboratory $z, x$ coordinate system are determined by the constraints that (i) the focal point of the rays $(z, x)$ $=(f a, 0)$ lies on the ellipse, (ii) the slope of the ellipse is zero where the incident ray enters the lens at $(z, x)$ $=(-a \cos (\beta), a \sin (\beta))$, and (iii) the path satisfies the ray propagation condition of Eqs. (4) and (5). After a reasonably large amount of algebra one obtains

$$
\begin{aligned}
\sin (2 \alpha) & =-\sin (2 \beta) / D, \\
\cos (2 \alpha) & =\left[f^{2}+\cos (2 \beta)\right] / D, \\
A & =\left(1+f^{2}+D\right) / 2, \\
B & =\left(1+f^{2}-D\right) / 2,
\end{aligned}
$$

where

$$
D=\left[f^{4}+2 f^{2} \cos (2 \beta)+1\right]^{1 / 2} .
$$

In Eqs. (20a) and (20b) care must be taken when approaching the limits $f \rightarrow 1$ and $\beta \rightarrow \pi / 2$ since $D \rightarrow 0$ there. The trajectory of a ray inside the lens in polar coordinates in the laboratory coordinate system is

$$
r(\xi)=2^{1 / 2} f a \sin (\beta) /\left[1+f^{2}-D \cos (2 \xi-2 \alpha)\right]^{1 / 2}
$$

for $\beta+2 \alpha \leqslant \xi \leqslant \pi-\beta$. The ray enters the sphere at $\xi=\pi-\beta$ and it exits at $\xi=\beta+2 \alpha$. The ray makes its closest approach to the origin halfway along the trajectory when 


$$
\xi_{0}=\pi / 2+\alpha
$$

and the distance of closest approach to the origin is

$$
r_{0}=r\left(\xi_{0}\right)=a B^{1 / 2} .
$$

\section{B. Scattering Angle and Scattered Intensity}

Since rays incident on the top half of the Luneburg lens focus at $(z, x)=(f a, 0)$ inside the lens, they exit on the bottom half of the lens where $\xi_{\text {exit }}<0$. Since Eqs. (3) and (5) require that

$$
\xi_{\text {exit }}=\beta+2 \alpha,
$$

the ray trajectories satisfy $|2 \alpha|>\beta$ for this geometry. The scattering angle can then be obtained from conservation of angular momentum and geometrical considerations, giving

$$
\theta=\beta-\xi_{\text {exit }}=-2 \alpha \text {. }
$$

This relation is graphed in Fig. 4. For future reference, Eqs. (20a) and (26) can be used to obtain

$$
\mathrm{d} \theta / \mathrm{d} \beta=2\left[1+f^{2} \cos (2 \beta)\right] / D^{2} .
$$

Since we are considering $f<1$ here, Eq. (27) gives $\mathrm{d} \theta / \mathrm{d} \beta$ $>0$. Thus $\theta$ is a monotonically increasing function of $\beta$. As is seen in Fig. 4 , as $\beta \rightarrow \pi / 2$ the scattering angle $\theta$ sharply increases toward $180^{\circ}$. This behavior is also evident in Fig. 2(b). For scattering of a plane wave by a homogeneous sphere, the scattering angle is standardly written [19] as a function of the angle of incidence $\beta$, and the relation $\theta(\beta)$ can be analytically inverted to give $\beta(\theta)$ only for external reflection and transmission. In like manner, Eqs. (20b) and (26) for transmission through a modified Luneburg lens can be inverted to obtain the angle of incidence $\beta$ as a function of the scattering angle $\theta$; i.e.,

$$
\cos (2 \beta)=\cos (\theta)\left[1-f^{4} \sin ^{2}(\theta)\right]^{1 / 2}-f^{2} \sin ^{2}(\theta) .
$$

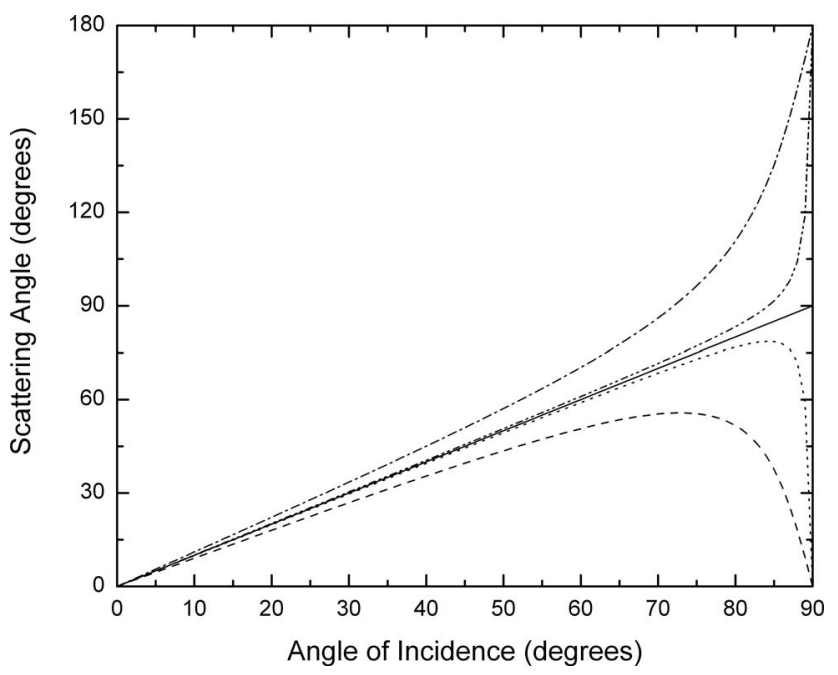

Fig. 4. Scattering angle $\theta$ as a function of the ray angle of incidence $\beta$ for $f=0.90$ (dot-dashed curve), $f=0.99$ (dot-dot-dashed curve), $f=1.0$ (solid curve), $f=1.01$ (dotted curve), and $f=1.10$ (dashed curve).
This analytical invertibility will have significant physical consequences in Section 5 for the case $f>1$. Again for future reference, one may write $D$ in terms of $\theta$ as

$$
D=f^{2} \cos (\theta)+\left[1-f^{4} \sin ^{2}(\theta)\right]^{1 / 2} .
$$

The scattered intensity can again be obtained from flux conservation in terms of $\theta$ alone using Eqs. (13) and (27) (29). One obtains

$$
\begin{aligned}
I_{\text {scat }}(\theta)= & \left(I_{0} a^{2} / 4 R^{2}\right)\left\{f^{2} \cos (\theta)\right. \\
& \left.+\left[1-f^{4} \sin ^{2}(\theta)\right]^{1 / 2}\right\}^{2} /\left[1-f^{4} \sin ^{2}(\theta)\right]^{1 / 2}
\end{aligned}
$$

for $0 \leqslant \theta \leqslant \pi$. One can now easily observe the nonuniform convergence of the scattering angle in the vicinity of $f=1$ and $\beta=\pi / 2$. First, it has already been shown that for $f$ $=1$ the scattering angle $\theta$ monotonically increases from 0 to $\pi / 2$ as $\beta$ increases from 0 to $\pi / 2$. But when $f<1$ Eqs. (20a), (20b), and (26) show that $\theta$ monotonically increases from 0 to $\pi$ as $\beta$ increases from 0 to $\pi / 2$. The central ray propagates straight through the modified Luneburg lens and gives $\theta=0$, and the edge ray traces out half of an ellipse inside the lens and is scattered with $\theta=\pi$. In addition, the derivative of the scattered electric field for $f=1$ diverged at $\theta=\pi / 2$. But for $f<1$, the intensity is smoothly attenuated in the vicinity of $\theta=\pi / 2$. Specifically, if $f=1$ $-\varepsilon$ with $\varepsilon \ll 1$, the scattered intensity at $\theta=\pi / 2$ is

$$
I_{\text {scat }}(\pi / 2)=I_{0} a^{2} \varepsilon^{1 / 2} /\left(2 R^{2}\right),
$$

and at $\theta=\pi$ it has decreased to

$$
I_{\text {scat }}(\pi)=I_{0} a^{2} \varepsilon^{2} / R^{2} .
$$

This behavior is apparent in Fig. 3 .

\section{Phase of the Scattered Light}

The calculation of the optical path length of a ray inside the modified Luneburg lens for $f<1$ is more complicated than for $f=1$. But fortunately the integrals can still be evaluated analytically. One obtains

$$
\begin{aligned}
\int N \mathrm{~d} s= & 2 a \int_{\xi_{\text {exit }}}^{\pi-\beta} \mathrm{d} \xi \sin (\beta)\left\{\left(1+f^{2}\right) /\left[1+f^{2}-D \cos (2 \xi-2 \alpha)\right]\right. \\
& \left.-2 f^{2} \sin ^{2}(\beta) /\left[1+f^{2}-D \cos (2 \xi-2 \alpha)\right]^{2}\right\} \\
= & {\left[a\left(1+f^{2}\right) / f\right]\left[\pi / 2-\arctan (M / P)^{1 / 2}\right]+a \cos (\beta), \quad(33) }
\end{aligned}
$$

where

$$
\begin{gathered}
M=D+f^{2}-1, \\
P=D-f^{2}+1 .
\end{gathered}
$$

The integrand of Eq. (33) was obtained using the same substitutions as were made in obtaining the integrand of Eq. (15). As a check of Eq. (33), the optical path length from the entrance plane to the focal point inside the lens is

$$
L=\left[1+\pi\left(1+f^{2}\right) / 4 f\right],
$$

which is again independent of $\beta$, producing constructive interference of all the rays at the focal point. Combining Eqs. (30) and (33), including the phase of the incoming ray from the lens entrance plane to the lens surface, the 
phase of the corresponding outgoing ray from the lens surface to the lens exit plane, subtracting off the phase of the reference ray, and adding in Fraunhofer diffraction of a plane wave by a sphere of radius $a$, one obtains

$$
\begin{aligned}
E_{\text {scat }}(\theta)= & \left(E_{0} a / 2 R\right) \exp (i \Phi)\left\{f^{2} \cos (\theta)\right. \\
& \left.+\left[1-f^{4} \sin ^{2}(\theta)\right]^{1 / 2}\right\} /\left[1-f^{4} \sin ^{2}(\theta)\right]^{1 / 4} \\
& +i\left(E_{0} a / R\right) x_{L}\left[J_{1}\left(x_{L} \theta\right) /\left(x_{L} \theta\right)\right] \exp (i k R),
\end{aligned}
$$

where

$$
\Phi=k R+x_{L}\left\{\left[\left(1+f^{2}\right) / f\right]\left[\pi / 2-\arctan (M / P)^{1 / 2}\right]-\cos (\beta)\right\}-\pi,
$$

and $\cos (\beta)$ can be obtained as a function of $\theta$ using Eq. (28).

\section{SCATTERING ANGLE, INTENSITY, AND PHASE FOR $\boldsymbol{f}>\mathbf{1}$}

For $f>1$, rays incident on the upper half of the lens also exit the lens above the $z$ axis, crossing the $z$ axis outside the lens and producing a near-zone transmission caustic, in contrast to the point focus for $f \leqslant 1$. This is suggested in Fig. 2(c). The parameters $A, B$, and $\alpha$ for the shape of the elliptical ray trajectory inside the lens are again given by Eqs. (20a)-(20d), the exit angle is again given by Eq. (25), and the scattering angle is again given by Eq. (26). Since Eqs. (21) and (27) dictate that $\mathrm{d} \theta / \mathrm{d} \beta<1$, the ray trajectories satisfy $|\alpha|<2 \beta$ for this geometry. In contrast to the case of $f=1$ where the grazing incidence ray traveled on the surface of the lens for a quarter revolution before breaking free, the grazing incidence ray now merely touches the surface of the lens and is scattered at $\theta=0$. This can be seen in Fig. 2(c) and in Fig. 4 where $\theta$ quickly decreases to zero as $\beta \rightarrow \pi / 2$. As a consequence, from Eq. (27), the rays transmitted through an $f>1$ modified Luneburg lens exhibit a rainbow [20], i.e., $\mathrm{d} \theta / \mathrm{d} \beta=0$, when

$$
\cos \left(2 \beta_{R}\right)=-1 / f^{2}
$$

corresponding to the Descartes rainbow scattering angle

$$
\sin \left(\theta_{R}\right)=1 / f^{2}
$$

Although a transmission rainbow cannot occur for a plane wave incident on a homogeneous sphere, it can occur when the high symmetry of that situation is broken in any one of a number of ways, such as when the sphere is distorted into a spheroid or when the incident wavefronts are converging rather than flat [21]. Allowing the sphere to be radially inhomogeneous is yet another way to break the symmetry. Other $N(r)$ profiles that give rise to interesting rainbow phenomena for one or more internal reflections have been investigated in [22,23]. Equations (20b) and (26), which express the scattering angle $\theta$ as a function of the impact parameter $\beta$, can again be analytically inverted to give

$$
\cos (2 \beta)=-f^{2} \sin ^{2}(\theta) \pm \cos (\theta)\left[1-f^{4} \sin ^{2}(\theta)\right]^{1 / 2},
$$

where the upper/lower sign corresponds to supernumerary rays with smaller/larger impact parameters. This has significant implications. Specifically, what one would ide- ally like to know in ray theory is the scattered intensity as a function of the scattering angle, i.e., $I_{\text {scat }}(\theta)$. But when $\theta(\beta)$ is not analytically invertible, as is the case for one internal reflection for scattering by a homogeneous sphere, one is forced to live with the inconvenient situation of having both $I_{\text {scat }}$ and $\theta$ being functions of $\beta$. One either has to obtain $\beta(\theta)$ numerically or approximate it in a small interval via a Taylor series expansion, and then substitute the result into $I_{\text {scat }}(\beta)$. For the $f>1$ modified Luneburg lens, the goal of obtaining $I_{\text {scat }}$ as a function of $\theta$ is achieved as a result of the analytical invertibility of $\theta(\beta)$ in Eq. (40). One obtains

$$
\begin{aligned}
I_{\text {scat }}(\theta)= & \left(I_{0} a^{2} / 4 R^{2}\right)\left\{f^{2} \cos (\theta)\right. \\
& \left.+\left[1-f^{4} \sin ^{2}(\theta)\right]^{1 / 2}\right\}^{2} /\left[1-f^{4} \sin ^{2}(\theta)\right]^{1 / 2} \\
& \text { for } 0 \leqslant \beta \leqslant \beta_{R}, \\
= & \left(I_{0} a^{2} / 4 R^{2}\right)\left\{f^{2} \cos (\theta)\right. \\
& \left.-\left[1-f^{4} \sin ^{2}(\theta)\right]^{1 / 2}\right\}^{2} /\left[1-f^{4} \sin ^{2}(\theta)\right]^{1 / 2} \\
& \text { for } \beta_{R} \leqslant \beta \leqslant \pi / 2 .
\end{aligned}
$$

The magnitude of the scattering amplitude $E_{ \pm}$is obtained by taking the square root of Eq. (41).

Again one can easily observe the nonuniform convergence of both the scattering angle and the scattered intensity in the vicinity of $f=1$ and $\beta=\pi / 2$. As is seen in Fig. 4, for $f<1$ the scattering angle of the $\beta=\pi / 2$ edge ray incident on the lens was $\pi$, for $f=1$ it was $\pi / 2$, and now for $f>1$ it is 0 . Also, if $f \gg 1$ the rainbow impact parameter $\beta_{R}$ is near $\pi / 4$ and the rainbow scattering angle $\theta_{R}$ is near 0 . But when $f$ decreases to $f=1+\varepsilon$ with $\varepsilon \ll 1$, the rainbow migrates to larger scattering angles. This is seen in Fig. 3, where for $f=1.10, \theta_{R}=55.74^{\circ}$ and for $f=1.01, \theta_{R}=78.61^{\circ} \mathrm{In}$ both cases the rays for $\beta>\beta_{R}$ rapidly damp to zero for $\theta$ $<\theta_{R}$, whereas the rays for $\beta<\beta_{R}$ produce an intensity as a function of $\theta$ that differs only minimally from the $f=1$ case. As $f \rightarrow 1$, the ray theory divergence of the intensity at the rainbow angle occurs at

$$
\theta_{R}=\pi / 2-2 \varepsilon^{1 / 2} .
$$

On the other hand, when the $f=1$ limit is taken first, the scattered intensity at $\theta=\pi / 2$ is zero.

In analogy to Eq. (33), the phase of the two supernumerary rays for $f>1$ is

$$
\Phi_{ \pm}=x_{L}\left\{\left[\left(1+f^{2}\right) / f\right]\left[\pi / 2-\arctan \left(M_{ \pm} / P_{ \pm}\right)^{1 / 2}\right]-\cos (\beta)\right\}+\zeta_{ \pm},
$$

where

$$
\begin{aligned}
& D_{ \pm}=f^{2} \cos (\theta) \pm\left[1-f^{4} \sin ^{2}(\theta)\right]^{1 / 2}, \\
& M_{ \pm}=D_{ \pm}+f^{2}-1 \\
& P_{ \pm}=D_{ \pm}-f^{2}+1
\end{aligned}
$$

and $\cos (\beta)$ can be obtained as a function of $\theta$ using Eq. (40). Again the upper/lower sign in Eqs. (43) and (44a)(44c) corresponds to the supernumerary ray with smaller/ 
larger impact parameter. In addition, the non-path-length phase is

$$
\begin{aligned}
\zeta_{ \pm} & =-\pi \quad \text { for } 0 \leqslant \beta<\beta_{R}, \\
& =-\pi / 2 \text { for } \beta_{R}<\beta \leqslant \pi / 2,
\end{aligned}
$$

using the van de Hulst rules [18] since the smaller impact parameter supernumerary rays both cross the $z$ axis and participate in the near-zone transmission caustic, whereas the larger impact parameter supernumerary rays cross the $z$ axis without participating in the transmission caustic.

By calculating the intersection coordinates of two rays with infinitesmally close incident impact parameters, the shape of the transmission caustic is found to be

$$
\begin{aligned}
& z / a=K \cos (2 \alpha) \cos (\beta)-\sin (2 \alpha) \sin (\beta), \\
& x / a=K \sin (2 \alpha) \cos (\beta)+\cos (2 \alpha) \sin (\beta),
\end{aligned}
$$

with

$$
K=D^{2} /\left\{2\left[1+f^{2} \cos (2 \beta)\right]\right\}
$$

and where $D$ is given as a function of $\beta$ in Eq. (21). The coordinates of the tip of the caustic are $x_{c}=0$ and $z_{c}$ $=a\left(1+f^{2}\right) / 2$, and as is suggested in Fig. 2(c), the caustic opens toward $+z$ rather than opening toward the lens as does the transmission caustic of a homogeneous sphere. The two curved arms of the caustic of Eqs. (46a) and (46b) evolve into the transmission rainbow and approach infinity as the scattering angle approaches $\theta_{R}$. In the vicinity of the paraxial focus, the shape of the caustic becomes the cusp:

$$
x= \pm\left[(4 / 3)\left(z-z_{c}\right)\right]^{3 / 2} /\left[\left(f^{2}-1\right)\left(f^{2}+1\right)^{1 / 2}\right] .
$$

\section{AIRY THEORY DESCRIPTION OF THE $f>1$ RAINBOW}

In this section the ray theory electric field

$$
E_{\text {scat }}(\theta)=E_{+} \exp \left(i \Phi_{+}\right)+E_{-} \exp \left(i \Phi_{-}\right)
$$

is obtained in the vicinity of the rainbow by starting with Eqs. (41) and (43) and expanding the magnitudes and phases in powers of $\Delta$, where

$$
\theta=\theta_{R}-\Delta
$$

and $\Delta \ll 1$, assuming the rainbow is not in the vicinity of $f=1$ and $\beta=\pi / 2$ where $D=0$. The first three nonzero terms of the Taylor series expansion of the phase $\Phi_{ \pm}$of Eq. (43) with respect to $\theta$ about the rainbow angle $\theta_{R}$ must be computed. It was found, however, to be easier to accomplish this by first writing $\Phi_{ \pm}$as a function of $\beta$, evaluating the derivatives of $\Phi_{ \pm}$with respect to $\beta$, and then substituting in $\beta-\beta_{R}$ as a function of $\theta-\theta_{R}$. These derivatives end up being relatively uncomplicated, e.g.,

$$
\mathrm{d} \Phi_{ \pm} / \mathrm{d} \beta=x_{L} \sin (\beta)\left[1-\left(f^{4}-1\right) / D^{2}\right],
$$

where $D$ is given as a function of $\beta$ in Eq. (21). The expansion of $\Phi_{ \pm}$is then

$$
\begin{aligned}
\Phi(\beta)= & \Phi_{R}-2^{1 / 2} x_{L}\left(\beta-\beta_{R}\right)^{2} /\left[f\left(f^{2}-1\right)^{1 / 2}\right] \\
& -2^{3 / 2} x_{L}\left(f^{2}+2\right)\left(\beta-\beta_{R}\right)^{3} /\left[3 f\left(f^{2}-1\right)\left(f^{2}+1\right)^{1 / 2}\right] \\
& +O\left[\left(\beta-\beta_{R}\right)^{4}\right],
\end{aligned}
$$

where $\Phi_{R}$ is the optical path length portion of (43) evaluated at the rainbow angle. Let

$\beta=\beta_{R}+\varepsilon \quad$ for the larger-impact-parameter

supernmerary ray,

$=\beta_{R}-\varepsilon \quad$ for the smaller-impact-parameter

supernumerary ray,

where $\varepsilon \ll 1$. Then starting from the expression for $\tan (\theta)$ given by Eqs. (20a), (20b), and (26), the relation between $\varepsilon$ and $\Delta$ is found to be

$$
\varepsilon=\left(f^{4}-1\right)^{1 / 4}(\Delta / 2)^{1 / 2} \pm \Delta / 2+O\left(\Delta^{3 / 2}\right) .
$$

Combining Eqs. (52)-(54) one finally obtains

$$
\Phi_{ \pm}(\theta)=\Phi_{R}-x_{L} \Delta \sin \left(\beta_{R}\right) \pm 2 x_{L} \Delta^{3 / 2} / 3 h^{1 / 2}+O\left(\Delta^{2}\right),
$$

where

$$
h=4 f^{2} /\left[\left(f^{2}-1\right)^{3 / 2}\left(f^{2}+1\right)^{1 / 2}\right] .
$$

The phase of Eq. (55) has the same form as does the oneinternal-reflection phase for scattering by a homogeneous sphere in the vicinity of the rainbow [24] except for the sign change of the second and third terms.

The first term in the Taylor series of the magnitudes $E_{ \pm}$ is obtained by expanding the square root of Eq. (41) in powers of $\Delta$ about the rainbow angle. The result is

$$
E_{ \pm}=\left(E_{0} a / 2 R\right)\left[\left(f^{4}-1\right)^{3 / 8} /(2 \Delta)^{1 / 4}\right]\left[1 \pm(2 \Delta)^{1 / 2} /\left(f^{4}-1\right)^{1 / 4}\right] .
$$

Combining the magnitudes and phases, defining

$$
u \equiv x_{L}^{2 / 3} \Delta / h^{1 / 3}
$$

and using the asymptotic forms of the Airy integral and its derivative deep in the two-ray region [25],

$$
\begin{gathered}
u^{1 / 4} \pi^{1 / 2} A i(-u) \rightarrow \sin \left[\left(2 u^{3 / 2} / 3\right)+\pi / 4\right], \\
-u^{-1 / 4} \pi^{1 / 2} A i^{\prime}(-u) \rightarrow \cos \left[\left(2 u^{3 / 2} / 3\right)+\pi / 4\right],
\end{gathered}
$$

one obtains

$$
\begin{aligned}
E_{\text {scat }}(\theta)= & \left(E_{0} / k R\right)\left[2^{3 / 4} \pi^{1 / 2}\left(f^{2}+1\right)^{5 / 12} /(2 f)^{1 / 6}\right] \\
& \times \exp \left[i\left(\Phi_{R}-x_{L} \Delta \sin \left(\beta_{R}\right)-3 \pi / 4\right)\right] \\
& \times\left\{\left(f^{2}-1\right)^{1 / 2} x_{L}^{7 / 6} A i(-u)\right. \\
& \left.+i 2^{5 / 6}\left[f /\left(f^{2}+1\right)\right]^{1 / 3} x_{L}^{5 / 6} A i^{\prime}(-u)\right\} .
\end{aligned}
$$

This result merits a number of comments. The usual way of deriving the Airy model of the one-internalreflection rainbow of a homogeneous sphere is to propagate the initially flat wavefront through the sphere, calculate the shape of the cubic wavefront at the sphere's exit plane at the rainbow scattering angle, and then Fraunhofer diffract it to the scattering far zone. This was not the method employed here to obtain Eq. (60). What 
was used instead was the fact that the asymptotic form of the Airy theory rainbow far into the illuminated region becomes the interference of two supernumerary rays. The two rays in the supernumerary region and their interference pattern were obtained first and then were used to work backward to obtain the electric field in the rainbow region in terms of the Airy integral and its derivative. This was also the procedure used in [26,27] to obtain the Airy description of the rainbow reflected from the neck region of a pendant droplet. The validity of this procedure was checked by applying it to the $p-1$ internal reflection rainbow of a homogeneous sphere. The results of both CAM theory [28] and ray theory for the transverse electric polarization agreed with the asymptotic limit of Airy theory, giving [29-31]

$$
\begin{aligned}
E_{\text {scat }}(\theta) \propto & C x_{H}^{7 / 6} A i(-u) \\
& +i\left\{\left[2 p^{2}-\left(9-p^{2}\right) S^{2}\right] /\left[6 p^{4 / 3}\left(p^{2}-1\right)^{1 / 3} S^{4 / 3}\right]\right\} \\
& \times x_{H}^{5 / 6} A i^{\prime}(-u)
\end{aligned}
$$

where

$$
\begin{aligned}
& S=\sin \left(\beta_{R}\right)=\left[\left(p^{2}-N^{2}\right) /\left(p^{2}-1\right)\right]^{1 / 2}, \\
& C=\cos \left(\beta_{R}\right)=\left[\left(N^{2}-1\right) /\left(p^{2}-1\right)\right]^{1 / 2},
\end{aligned}
$$

and $x_{H}$ is the size parameter of the homogeneous sphere in analogy to Eq. (19) for the Luneburg lens. For scattering by a homogeneous sphere, the $x_{H}^{7 / 6}$ dependence of the scattered intensity is associated with $A i(-u)$ rather than $A i^{\prime}(-u)$ because the larger-impact-parameter supernumerary ray has a larger amplitude, a longer optical path length, and a more negative non-path-length phase than does the smaller-impact-parameter supernumerary ray. The situation for the transmission rainbow of a modified Luneburg lens is the opposite of this. The $A i(-u)$ term is again proportional to $x_{L}^{7 / 6}$ because the larger-impactparameter supernumerary ray has a smaller amplitude, a shorter optical path length, and a more positive non-pathlength phase than does the smaller-impact-parameter supernumerary ray.

As $f$ decreases but does not get too close to the nonuniform convergence point at $f=1$, the dominant dependence of the rainbow of Eq. (60) shifts from the $x_{L}^{7 / 6} A i(-u)$ factor to the $x_{L}^{5 / 6} A i^{\prime}(-u)$ factor. As a result the rainbow's peak intensity becomes less pronounced as a function of $x_{L}$ and its supernumerary interference pattern becomes more pronounced [32]. This is also the case for the TE polarization for the $p-1$ internal reflection rainbow of a homogeneous sphere in the $N \rightarrow 1$ limit and for the TM polarization of the $p=2$ internal reflection rainbow [33] for $N$ $\approx 1.33$. Even though the Taylor series expansions involved in the calculations leading to Eq. (60) are not valid in the strict $f \rightarrow 1$ limit, the growing supernumerary interference pattern for $\theta<\theta_{R}$ will be seen in $[9,10]$ to evolve into the oscillatory portion of the Fresnel straight-edge diffraction pattern [17] produced by the orbiting ray when $f=1$. Similarly, the rainbow's complex ray contribution for $\theta>\theta_{R}$, which in wave theory is on the classically forbidden side of the rainbow and smoothes the rainbow's transition from the illuminated region to the shadowed region, will be seen to evolve into the monotonically decreasing portion of the Fresnel straight-edge diffraction pattern produced by the orbiting ray when $f=1$. Last, the decrease in the height of the main rainbow peak serves to smoothly bridge these two limiting behaviors.

\section{CONCLUSIONS}

Both the Luneburg lens and the modified Luneburg lens provide interesting and analytically soluble prototype examples of scattering of a plane wave by a sphere with a radially inhomogeneous refractive index profile. They have features in common with scattering by a homogeneous sphere. They also have new features that are due to the fact that the ray paths inside the sphere are curved and that demonstrate the richness of phenomena encountered in electromagnetic scattering. An intriguing scattering phenomenon of a Luneburg lens described in part here and further pursued in $[9,10]$ is the evolution of the transmission rainbow for $f>1$ to the orbiting ray for $f=1$ to families of MDRs for $f<1$ and the associated nonuniform convergence of both the ray intensity and ray trajectories inside the lens in the vicinity of this transition. The way in which the divergences and discontinuities of ray theory are smoothed by the diffractive behavior of light waves will be derived and interpreted in the remaining two papers in this series [34].

\section{REFERENCES AND NOTES}

1. M. Born and E. Wolf, Principles of Optics, 6th ed. (Cambridge U. Press, 1980), footnote p. 147.

2. H. Sakurai, T. Hashidate, M. Ohki, K. Motojima, and S. Kozaki, "Electromagnetic scattering by the Luneburg lens reflector," Int. J. Electron. 84, 635-645 (1998).

3. A. D. Greenwood and J.-M. Jin, "A field picture of wave propagation in inhomogeneous dielectric lenses," IEEE Antennas Propag. Mag. 41, 9-17 (1999).

4. J. M. Gordon, "Spherical gradient-index lenses as perfect imaging and maximum power transfer devices," Appl. Opt. 39, 3825-3832 (2000).

5. A. S. Gutman, "Modified Luneburg lens," J. Appl. Phys. 25, 855-859 (1954).

6. J. Sochacki, "Exact analytical solution of the generalized Luneburg lens problem," J. Opt. Soc. Am. 73, 789-795 (1983).

7. J. Sochacki, "Generalized Luneburg lens problem solution: a comment," J. Opt. Soc. Am. 73, 1839 (1983).

8. J. R. Flores, J. Sochacki, M. Sochacka, and R. Staronski, "Quasi-analytical ray tracing through the generalized Luneburg lens," Appl. Opt. 31, 5167-5170 (1992).

9. J. A. Lock, "Scattering of a plane wave by a Luneburg lens. II. Wave theory," J. Opt. Soc. Am. A 25, 2980-2990 (2008).

10. J. A. Lock, "Scattering of a plane wave by a Luneburg lens. III. Finely stratified multilayer sphere model," J. Opt. Soc. Am. A 25, 2991-3000 (2008).

11. K. W. Ford and J. A. Wheeler, "Semiclassical description of scattering," Ann. Phys. (N.Y.) 7, 259-286 (1959).

12. M. V. Berry and K. E. Mount, "Semiclassical approximations in wave mechanics," Rep. Prog. Phys. 35, 315-397 (1972), Secs. 6.2, 6.3.

13. M. Born and E. Wolf, Principles of Optics, 6th ed. (Cambridge U. Press, 1980), p. 123.

14. B. R. Johnson, "Light scattering by a multilayer sphere," Appl. Opt. 35, 3286-3296 (1996).

15. H. C. van de Hulst, Light Scattering by Small Particles (Dover, 1981), p. 205.

16. C. F. Bohren and D. R. Huffman, Absorption and Scattering of Light by Small Particles (Wiley-Interscience, 1983), p. 167. 
17. N. Fiedler-Ferrari, H. M. Nussenzveig, and W. J. Wiscombe, "Theory of near-critical-angle scattering from a curved interface," Phys. Rev. A 43, 1005-1038 (1991).

18. H. C. van de Hulst, Light Scattering by Small Particles (Dover, 1981), p. 207.

19. C. F. Bohren and D. R. Huffman, Absorption and Scattering of Light by Small Particles (Wiley-Interscience, 1983), p. 175 .

20. F. Michel, G. Reidemeister, and S. Ohkubo, "Luneburg lens approach to nuclear rainbow scattering," Phys. Rev. Lett. 89, 152701-1-152701-4 (2002).

21. J. A. Lock and T. A. McCollum, "Further thoughts on Newton's zero-order rainbow,” Am. J. Phys. 62, 1082-1089 (1994).

22. C. L. Brockman and N. G. Alexopoulos, "Geometrical optics of inhomogeneous particles: glory ray and the rainbow revisited," Appl. Opt. 16, 166-174 (1977).

23. J. A. Adam and P. Laven, "Rainbows from inhomogeneous transparent spheres: a ray-theoretic approach," Appl. Opt. 46, 922-929 (2007).

24. H. M. Nussenzveig, "High-frequency scattering by a transparent sphere. II. Theory of the rainbow and the glory," J. Math. Phys. 10, 125-176 (1969).

25. M. Abramowitz and I. A. Stegun, Handbook of Mathematical Functions (National Bureau of Standards, 1964), pp. 448, 449 and Eqs. (10.4.60), (10.4.62).

26. J. A. Lock, C. L. Adler, and R. F. Fleet, "Rainbows in the grass I: external-reflection rainbows from pendant droplets," Appl. Opt. (to be published).
27. C. L. Adler, J. A. Lock, and R. F. Fleet, "Rainbows in the grass II: arbitrary diagonal incidence," Appl. Opt. (to be published).

28. V. Khare and H. M. Nussenzveig, "Theory of the rainbow," Phys. Rev. Lett. 33, 976-980 (1974).

29. There are some discrepancies in the literature concerning Eq. (61). The form given here when applied to $p=2$ and $N$ $=1.333$ agrees with Eq. (5) and Table 1 of [28] but does not agree for $p=2$ and $N=1.333$ with Eqs. (4.30), (4.31), and (4.34) of the earlier [24], which were reproduced as Eqs. (5.106) and (5.108) of [30]. The appropriate quantities to expand in Taylor series about the rainbow angle are Eq. (4.29) of [24] and Eq. (5.62) of [31].

30. W. T. Grandy, Scattering of Waves from Large Spheres (Cambridge U. Press, 2000).

31. J. A. Adam, "The mathematical physics of rainbows and glories," Phys. Rep. 356, 229-365 (2002).

32. M. Abramowitz and I. A. Stegun, Handbook of Mathematical Functions (National Bureau of Standards, 1964), p. 446, Fig. 10.6.

33. G. P. Konnen and J. H. de Boer, "Polarized rainbow," Appl. Opt. 18, 1961-1965 (1979).

34. Most of the results of this paper and of $[9,10]$ were presented in preliminary form at the Fall Meeting of the Ohio Section of the American Physical Society, Cleveland Ohio, October 14-15, 2005. 